\title{
Mining the Twittersphere: Insights about Public Interest in Facial Reanimation Surgery from a Decade of Twitter Data
}

\author{
Hayeem L. Rudy ${ }^{1}$ Joseph A. Ricci ${ }^{1}$ \\ ${ }^{1}$ The Division of Plastic and Reconstructive Surgery, Department of \\ Surgery, Montefiore Medical Center, Bronx, New York, United States \\ Indian J Plast Surg 2022;55:31-35.
}

Address for correspondence Joseph A. Ricci, MD, Division of Plastic and Reconstructive Surgery, Montefiore Medical Center, 1776 Eastchester Road, Suite 200, Bronx, NY 10461, United States (e-mail: Dr.joseph.ricci@gmail.com).

Background With 500 million tweets posted daily, Twitter can provide valuable insights about public discourse surrounding niche topics, such as facial paralysis surgery. This study aims to describe public interest on Twitter relating to facial paralysis and facial reanimation surgery over the last decade.

Methods Tweets containing the keywords "facial paralysis" and "Bell's palsy" posted between January 1, 2009, and January 1, 2019, were collected using Twitter Scraper. Tweets were screened by keywords relating to facial paralysis, and usage of these terms trended over time. Logistic regression was used to identify correlations between the quantity of publications per year and these terms posted on Twitter.

Results 32,880 tweets were made during the study period, with no significant difference in the number of tweets per year. A very strong $(r=0.8-1.0)$ positive correlation was found between time and frequency of the term "plastic surgery" and "transfer" $(p<0.05)$. A strong $(r=0.60-0.79)$ correlation was found between time and frequency for the following terms: "facial reanimation," "gracilis," "masseter," "plastics," "transplant" $(p<0.05)$. A total of 619 studies with the keyword "facial reanimation" were published in PubMed within the study period. A very strong, positive correlation between publications per year and frequency was found for the terms "plastic surgery," "function" and "esthetic," and a strong, positive correlation was found for the "plastics," "transplant," "Botox," "surgery," "cosmetic," "aesthetic" and "injection" $(p<0.05)$.

Conclusions An increasing number of discussion about facial paralysis on Twitter correlates with increased publications and likely surgeon discourse on facial reanimation surgery, driving public interest.

\section{Introduction}

Social media has become increasingly important in plastic surgery. ${ }^{1-3}$ In the last decade, surgeons and patients have started to use social media as a tool for marketing and engaging in dialogue about plastic surgery. ${ }^{4-7}$ Prior research has investigated the top plastic surgery "influencers" on Twitter, the veracity of information about plastic surgery published online December 13, 2021
DOI https://doi.org/ $10.1055 / \mathrm{s}-0041-1740080$. ISSN 0970-0358. (c) 2021. Association of Plastic Surgeons of India. All rights reserved. This is an open access article published by Thieme under the terms of the Creative Commons Attribution-NonDerivative-NonCommercial-License, permitting copying and reproduction so long as the original work is given appropriate credit. Contents may not be used for commercial purposes, or adapted, remixed, transformed or built upon. (https://creativecommons.org/ licenses/by-nc-nd/4.0/)

Thieme Medical and Scientific Publishers Pvt. Ltd., A-12, 2nd Floor, Sector 2, Noida-201301 UP, India 
published on YouTube, and broad characterizations of the type of dialogue about plastic surgery occurring over Twitter. ${ }^{8-11}$ While these studies represent early ventures into the area of social media data analyses, there is an untapped opportunity to leverage the massive amounts of data published on these platforms to gain insight into the public's understanding and interest in plastic surgery. To date, largescale data mining from social medial has not been attempted before to identify trends in public interest in various areas of plastic surgery.

One of the most relevant social media platforms in existence today is Twitter (Twitter, Inc.; San Francisco, CA), which receives over 500 million tweets daily on a global basis. ${ }^{10}$ When compared with other available social media platforms, Twitter is ideal for the real-time analysis of public interest in a specific topic for several reasons. First, unlike other platforms, Twitter provides an advanced programming interface (API), which makes it possible to pull datasets representing Twitter activity easily and free of charge. ${ }^{12}$ Second, because the majority of Twitter accounts are public, researchers are able to download tweets and data from the majority of users on the platform. Third, prior studies have shown that Twitter content relating to plastic surgery largely comprises tweets from patients, not surgeons or marketers, posting about their personal experience with or interest in plastic surgery procedures. ${ }^{10,11}$ As a result, Twitter offers a direct window into the public dialogue occurring over social media. Nonetheless, to date, few studies in the plastic surgery literature have attempted to utilize Twitter data for the purpose of understanding public trends.

This study aims to use Twitter to investigate trends in public dialogue relating to specific niche plastic surgery topics and procedure, which were previously never performed. In this case, the topic of facial reanimation surgery was chosen, given that it is a small but growing area within plastic and reconstructive surgery, about which current public interest and awareness is unknown, not having previously been studied. Given the annual incidence of facial paralysis in the US, the authors hypothesized that enough individuals would be discussing their experience with facial paralysis to be captured well on Twitter, but missed by other social media platforms, which would result in an adequately powered analysis. The purpose of this article was therefore to investigate public interest in and awareness of facial reanimation surgery by analyzing trends in the past decade of Twitter data relating to facial paralysis and facial reanimation surgery.

\section{Methods}

Twitter-scraper, a free and publicly accessible pythonbased program for aggregating Twitter data, was used to identify all included. ${ }^{13}$ The program identified and returned all posted in the US between January 1, 2009, and January 1, 2019, which contained either of the key words "facial paralysis" or "Bell's palsy." The program returned the following data for each tweet: content, post
Table 1 List of terms relating to reconstructive techniques for facial paralysis and facial reanimation surgery

\begin{tabular}{|l|l|l|l|}
\hline Reconstructive & Technique & Microsurgery & Esthetic \\
\hline Plastic surgery & Acupuncture & Transfer & Function \\
\hline Gracilis & Surgical Treatment & Reconstruction & $\begin{array}{l}\text { Facial } \\
\text { Reanimation }\end{array}$ \\
\hline Muscle transfer & Surgical & Study & Reanimate \\
\hline Nerve transfer & Plastics & Paper & Transplant \\
\hline Masseter & Cosmetic & Article & Moebius \\
\hline Reanimation & Aesthetic & Sling & Weight \\
\hline Botox & Reconstruction & Suspension & \\
\hline Surgery & Injection & Neurotization & \\
\hline Nerve surgery & Botulinum & Free muscle & \\
\hline
\end{tabular}

date, username, associated hashtags, number of retweets, and number of likes. Raw output data was imported into a CSV file using NumPy and subsequently organized in Microsoft Excel (Microsoft Inc., Redmond, Washington, USA). ${ }^{14}$

Prior to analysis, duplicate tweets were removed, and tweets were organized by date. A list of terms relating to facial reanimation surgery was generated from a literature search of recent review articles discussing facial reanimation surgery, which were posted on PubMed in the past 10 years. - Table 1 lists all terms that were included in this list. To determine whether the tweets were related to facial reanimation surgery or facial paralysis, the content for each tweet identified by the program was parsed using Microsoft Excel to identify whether it contained any of the identified key terms relating to facial reanimation surgery. Each time a reconstruction-related term appeared in a unique tweet, it was tabulated. After all tabulation was performed, the usage of reconstruction related terms were analyzed for trends in usage over time.

To determine the impact of research in facial reanimation surgery specifically on Twitter dialogue over the course of the study period, PubMed was indexed to identify the number of articles published within each year of the study period, which was associated with the keyword "facial reanimation." The number of published articles was then compared with the usage of reconstruction-related terms on Twitter in the same year. Multiple logistic regression was performed to determine the presence of trends in the frequency of term usage per year of the study period and to investigate the relationship between research activity in facial reanimation surgery and the usage of terms related to facial reanimation surgery each year. A very strong trend was defined as a correlation coefficient " $r$ " $=0.8-1.0$. A strong trend defined as " $r$ " $=0.60$ to 0.79 and a weak trend was defined as any " $r$ " value less than 0.60 .

\section{Results}

A total of 32,880 tweets that satisfied the Twitter-scraper criteria were posted to Twitter during the 10-year study 
My bby sister has a facial reanimation surgery tomorrow to help her facial paralysis. Extra positive vibes \& prayers are so appreciated

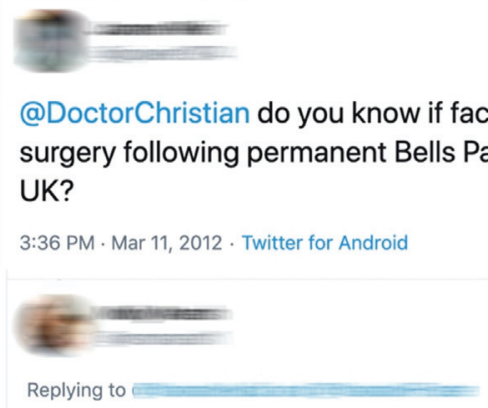

Thank you!l'm currently treating Lyme and Babesia. Suffered facial nerve paralysis requiring facial nerve transplant bc of misdiagnosis.

10:54 PM · Sep 13, 2017 - Twitter for iPad
Feeling a bit sick... waiting to have Botox injections! It's medicinal for my bells palsy. The thought of the needle makes me feel sick!

6:10 PM - Oct 24, $2010 \cdot$ TweetDeck

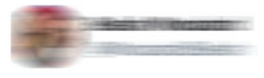

\#health3113 A recent study published in @JAMAFacial shows how Johns Hopkins plastic surgeon Kofi Boahene \& colleagues developed a multivector gracilis muscle flap to improve \#FacialParalysis outcomes.

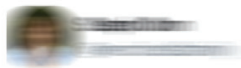

Replying to

I got bells palsy when i was 31, i am now 46 and $\mathrm{i}$ have never recovered, i have to have botox injections ever 3 months

7:43 PM · Jul 27, 2017 - Twitter Web Client

Fig. 1 Sample of publicly available tweets that were aggregated in the study and which included the usage of reconstruction-related terms. Account information blurred for privacy.

period. Examples of publicly available tweets discussing facial reanimation surgeries are demonstrated in -Fig. 1. The mean number of tweets per year was 3,288 , and there was no significant difference in the number of overall tweets posted per year of the study period ( $p=0.47$ ). Analysis of the use of reconstruction-related terms in these tweets showed significant trend toward increasing usage throughout the course of the study period. A very strong $(r=0.8-1.0)$ positive trend over time was found for the term "plastic surgery" $(p<0.05)$. A strong $(r=0.60-0.79)$ positive trend in frequency was found for the following terms: "facial reanimation," "gracilis," "masseter," "plastics," "transplant," and "transfer" $(p<0.05)$ (-Table 2). The remaining search terms demonstrated a weak or no correlation over time. - Fig. 2 shows the correlation score of all significantly associated reconstruction-related terms that were analyzed for trends in usage over time.

A total of 619 articles associated with the keyword "facial reanimation surgery" were published during the study period from 2009 to 2019. The mean number of articles published per year was 61.9. There were no significant differences in the number of articles published per year $(p=0.58)$. Regression analysis demonstrated a significant association between the number of studies published per year and the usage of reconstruction-related terms on Twitter in the same year. A very strong $(r=0.8-1.0)$ positive correlation was found for the following terms: "plastic surgery," "function," and "esthetic" $(p<0.05)$. A strong $(r=0.60-0.79)$ correlation was found for the following terms: "plastics," "transplant," "Botox," "surgery," "cosmetic," "aesthetic," and "injection" $(p<0.05)(-$ Table 3$)$. The remaining search terms yielded a weak correlation/no correlation.
Table 2 List of terms relating to reconstructive techniques that demonstrated statistically significant increase in usage on Twitter over a 10-year period

\begin{tabular}{|l|l|l|l|}
\hline Term & $r$-value & $p$-value & $\begin{array}{l}\text { Correlation } \\
\text { strength }\end{array}$ \\
\hline Plastic surgery & 0.81 & $<0.01$ & Very strong \\
\hline Gracilis & 0.75 & $<0.01$ & Strong \\
\hline Masseter & 0.65 & 0.04 & Strong \\
\hline Facial reanimation & 0.77 & $<0.01$ & Strong \\
\hline Plastics & 0.75 & $<0.01$ & Strong \\
\hline Injection & 0.84 & $<0.01$ & Very strong \\
\hline Transplant & 0.72 & 0.02 & Strong \\
\hline
\end{tabular}

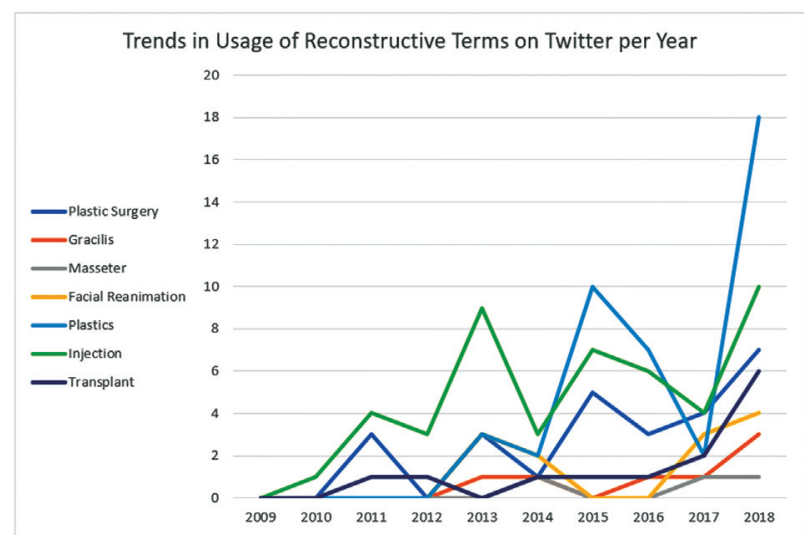

Fig. 2 Graph demonstrating the usage of reconstruction related terms over time on Twitter for terms that demonstrated a significant positive trend over the course of the study period. 
Table 3 List of terms that demonstrated statistically significant correlation between annual usage and number of studies published relating to facial reanimation surgery in the same year

\begin{tabular}{|l|l|l|}
\hline Term & $r$-value & $p$-value \\
\hline Esthetic & 0.8318 & $<0.01$ \\
\hline Function & 0.82669 & $<0.01$ \\
\hline Plastic surgery & 0.80609 & $<0.01$ \\
\hline Aesthetic & 0.78993 & $<0.01$ \\
\hline Botox & 0.74481 & 0.01 \\
\hline Surgery & 0.69937 & 0.02 \\
\hline Study & 0.68655 & 0.03 \\
\hline Injection & 0.682 & 0.03 \\
\hline Cosmetic & 0.67815 & 0.03 \\
\hline Transplant & 0.61823 & 0.05 \\
\hline
\end{tabular}

\section{Discussion}

Previously, researchers have used Google Trends, a free webbased tool for analyzing search queries over time, to investigate interest in plastic surgery procedures. For example, Tijerina and Whipple demonstrated a significant correlation between the annual number of Google search queries for various procedures and the annual surgical volumes for highvolume plastic surgery procedures from statistical reports of the American Society of Plastic Surgeons (ASPS) and American Society of Aesthetic Plastic Surgery (ASAPS). ${ }^{15-20}$

While demonstrating utility in investigating public interest in these broad areas of plastic surgery, Google Trends was not a well-suited tool to determine public interest in smaller, niche areas of plastic surgery such as facial reanimation. Rather than reporting the actual number of search queries per year for a given search term, Google Trends provides a score from 0 to 100 , which represents the relative popularity of a given search term when compared with all other terms searched over a specific time range. The popularity of other commonly searched terms dwarfs smaller topics, even within the scope of plastic surgery, making it difficult or near impossible to identify trends among these less researched topics. Performing the current study with a Google Trends search rather than a Twitter posts, returned a value of zero for the search term "facial reanimation surgery" across all years of the study period, despite the absolute values for search queries likely being greater than 0 . The same was true for Google Trend searches of the keywords that were used for Twitter queries in this study.

Twitter also provides access to a huge amount of raw data in the form of tweets that contain a certain keyword or hashtags, which can be downloaded along with associated data and analyzed independently, making it suitable for analysis of public opinion and interest in more niche topics. A recent study demonstrated that over $70 \%$ of tweets posted relating to plastic surgery are posted by the public, and not by promotional, professional or plastic surgeon accounts, which underscores the validity of using Twitter discourse as a representation of general public interests. ${ }^{10}$ Although limited analysis of Twitter has been performed to date, approximately 70 to $75 \%$ of tweets relating to plastic surgery are about cosmetic surgery. ${ }^{21}$ Despite this, the authors did, in fact, find significant trends in tweets relating to facial reanimation surgery, a niche topic even within reconstructive plastic surgery. For most surgeons, Twitter analyses can be useful both for monitoring the emergence of new procedures and ideas in the consumer marketplace and for making informed marketing decisions, based on public trends. Given the ease of use of Twitter's API, keywords relating to procedures can be monitored for trends in use overtime with little difficulty and generate meaningful insights in a cost-effective fashion. Furthermore, Twitter's API parameters allow for geographic localization of results, which can be used to identity regional trends that might be more relevant to individual practitioners, based on the location of their practice.

Finally, the authors hypothesize that public excitement about new treatments for facial reanimation would be a driver of this topic of conversation on Twitter, as tweets initially made from professional accounts would be read by and discussed by the public. This theory is consistent with prior studies of Twitter which have demonstrated that approximately 20 to $40 \%$ of tweets relating to plastic surgery, which were made by professionals, were research or education oriented. ${ }^{10,11}$ The present study did identify a significant association between the amount of research productivity per year and the annual amount of Twitter discourse relating to facial reanimation surgery. This could potentially represent that tweets by professionals (citing research to emphasize new techniques in facial reanimation surgery) might drive public excitement and therefore Twitter discourse on these topic.

There are several limitations to this study, including sampling bias and Twitter noise that are inherent to the study design. Recent data has demonstrated that while approximately $20 \%$ of Americans use Twitter, the platform is primarily used by younger demographics. ${ }^{22}$ Although facial paralysis affects patients of all ages, it is likely that the conversation on Twitter captured in this study underestimates the general interests of the overall public or is skewed toward the opinions of younger patients. Another challenge inherent to Twitter analyses is the presence of Twitter noise, which is the phenomenon of tweets unrelated to the subject being included in the analysis, because they contain the specified keywords being investigated in the study. Finally, our study included the use of terms that would be more commonly used in academic research articles (i.e., masseter, gracilis) and those that would be more common among the public (i.e., transplant). Therefore, our results likely captured both professional and nonprofessional Twitter users, the majority of whom were likely nonprofessional. Parsing through these users to include only nonprofessional users posed a technical challenge to the study that could not be overcome. 


\section{Conclusion}

Twitter offers access to robust datasets, which are helpful to determine trends in the public interest in niche topics in surgery. In the last decade, there has been an increasing discussion about facial paralysis on Twitter, which correlated with increased publications on facial reanimation surgery, implying that research in this area may be a driver of public discourse. Additionally, given the number of users, active surgeon participation in Twitter can potentially help to raise the public's awareness of this important problem for patients.

\section{Author Contributions}

H.R. designed the project, collected data, and drafted the manuscript.

J.R. designed the project, drafted the manuscript, edited the manuscript, and provided project oversight.

\section{Financial Disclosure Statement}

The authors have no financial disclosures. This work was not supported by any external sources of funding.

\section{Conflicts of Interest}

The authors have no conflicts of interest to report.

\section{References}

1 Sorice SC, Li AY, Gilstrap J, Canales FL, Furnas HJ. Social media and the plastic surgery patient. Plast Reconstr Surg 2017;140(05): 1047-1056

2 Liu DZ. Commentary on: A primer on social media for plastic surgeons: What do i need to know about social media and how can it help my practice? Aesthet Surg J 2017;37(05):620-621

3 Rohrich RJ, Savetsky IL, Savetsky EB, Avashia YJ. Why social media is transforming plastic surgery. Indian J Plast Surg 2020;53(01):4-5

4 Chandawarkar AA, Gould DJ, Stevens WG. Insta-grated plastic surgery residencies: the rise of social media use by trainees and responsible guidelines for use. Aesthet Surg J 2018;38(10): $1145-1152$

5 Reissis D, Shiatis A, Nikkhah D. Advertising on social media: the plastic surgeon's prerogative. Aesthet Surg J 2017;37(01): NP1-NP2

6 Ben Naftali Y, Duek OS, Rafaeli S, Ullmann Y. Plastic surgery faces the web: Analysis of the popular social media for plastic surgeons. Plast Reconstr Surg Glob Open 2018;6(12):e1958
7 Kadam D. Pandemic pause: a mixed blessing. Indian J Plast Surg 2020;53(02):169-170

8 Chandawarkar AA, Gould DJ, Grant Stevens W. The top 100 social media influencers in plastic surgery on twitter: who should you be following? Aesthet Surg J 2018;38(08):913-917

9 Gray MC, Gemmiti A, Ata A, et al. Can you trust what you watch? An assessment of the quality of information in aesthetic surgery videos on YouTube. Plast Reconstr Surg 2020;145(02): 329e-336e

10 Branford OA, Kamali P, Rohrich RJ, et al. \#PlasticSurgery. Plast Reconstr Surg 2016;138:1354-1365

11 Kalandar A, Al-Youha S, Al-Halabi B, Williams J. What does the public think? Examining plastic surgery perceptions through the twitterverse. Plast Reconstr Surg 2018;142(01):265-274

12 Developer. Accessed June 8, 2020 at: https://developer. twitter.com/en

13 Taspinar A. Twitter Scraper. Accessed May 24, 2021 at: https:// github.com/taspinar/twitterscraper

14 Millman KJ, Aivazis M. Python for scientists and engineers. Comput Sci Eng 2011;13(02):9-12

15 Tijerina JD, Morrison SD, Nolan IT, Parham MJ, Nazerali R. Predicting public interest in nonsurgical cosmetic procedures using Google Trends. Aesthet Surg J 2019 (e-pub ahead of print) Doi: $10.1093 / a s j / s j z 264$

16 Tijerina JD, Morrison SD, Vail DG, Lee GK, Nazerali R. The Utility of Google Trends Data for Analyzing Public Interest in Breast Procedures. Ann Plast Surg 2019;82(5S, Suppl 4)S325-S331

17 Tijerina JD, Morrison SD, Nolan IT, Vail DG, Lee GK, Nazerali R. Analysis and interpretation of Google Trends data on public interest in cosmetic body procedures. Aesthet Surg J 2020;40 (01):NP34-NP43

18 Tijerina JD, Morrison SD, Nolan IT, Vail DG, Nazerali R, Lee GK. Google Trends as a tool for evaluating public interest in facial cosmetic procedures. Aesthet Surg J 2019;39(08):908-918

19 Tijerina JD, Morrison SD, Nolan IT, Parham MJ, Richardson MT, Nazerali R. Celebrity influence affecting public interest in plastic surgery procedures: Google Trends analysis. Aesthetic Plast Surg 2019;43(06):1669-1680

20 Whipple LA, Kotamarti VS, Heiman AJ, Patel A, Ricci JA. Using Google Trends to analyze patient search interest in implantbased and autologous breast reconstruction. Breast J 2019; $00: 1-3$

21 Chopan M, Sayadi L, Clark EM, Maguire K. Plastic surgery and social media: examining perceptions. Plast Reconstr Surg 2019; 143(04):1259-1265

22 Demographics of Social Media Users and Adoption in the United States | Pew Research Center. Accessed April 13, 2020 at: https:/| www.pewresearch.org/internet/fact-sheet/social-media/ \#which-social-media-platforms-are-most-popular 